\title{
Storm-time magnetic field variations observed by the ETS-VI satellite
}

\author{
N. Terada ${ }^{1}$, T. Iyemori ${ }^{1}$, M. Nosé ${ }^{1}$, T. Nagai ${ }^{2}$, H. Matsumoto ${ }^{3}$ and T. Goka ${ }^{3}$ \\ ${ }^{1}$ Faculty of Science, Kyoto University, Kyoto 606-8502, Japan \\ ${ }^{2}$ Earth and Planetary Sciences, Tokyo Institute of Technology, Tokyo 152-8551, Japan \\ ${ }^{3}$ Office of Research and Development, National Space Development Agency of Japan, Tsukuba 305-0047, Japan
}

(Received September 5, 1997; Revised April 20, 1998; Accepted June 15, 1998)

\begin{abstract}
To study the ring current structure in the inner magnetosphere, we have statistically examined the magnetic field data acquired by ETS-VI (the Engineering Test Satellite-VI). During a magnetic storm, the Dst index shows a rapid recovery of its amplitude for about 9 hours on average after the main phase and a subsequent long-lasting slow recovery. We have investigated this "two-step recovery" of the Dst index by obtaining magnetic field vectors and calculating the current structure in the inner magnetosphere for each magnetic storm phase determined by the $D s t$ index. From this study, following results are obtained: (1) Throughout the storm-time, disturbed magnetic fields exhibit clear day-night asymmetry with strong peak in the nightside. (2) During the main phase, southward perturbed field components have a relative bump in the nightside region between $\sim 2000$ and $\sim 0400$ MLT and between $\sim 4.0$ and 6.4 $\mathrm{R}_{\mathrm{E}}$ (geocentric distances in Earth radii). (3) The initial rapid recovery of the Dst index is considerably influenced by the nightside currents flowing between $\sim 1800$ and $\sim 0600$ MLT and between 5.6 and 7.2 $\mathrm{R}_{\mathrm{E}}$. These currents are thought to be mainly composed of the particles that escape the magnetosphere on the duskside flank, which are simulated in particle tracing in a realistic magnetosphere.
\end{abstract}

\section{Introduction}

One of the most pronounced features during a magnetic storm is the growth and recovery of the ring current flowing in the inner magnetosphere. When a magnetic storm occurs, charged particles in the magnetotail are injected by an increase of the dawn-dusk electric field and form the ring current flowing mainly in the westward direction. The Dst index, which indicates ground level worldwide deviations of the southward geomagnetic field at middle and low latitudes, shows steep development by the storm-associated growth of this westward ring current and other magnetospheric currents such as the cross-tail current. It recovers rapidly in general after the main phase, and a subsequent gradual recovery follows (Fig. 1). This characteristic, so called "two-step recovery" of the Dst index, has been attributed to various loss mechanisms such as the multistep loss of charged particles caused by the difference of charge exchange lifetimes for the components of the closed ring current (e.g., Akasofu et al., 1963; Hamilton et al., 1988), the ion precipitation losses and so on. Hamilton et al. (1988) showed on the basis of the AMPTE/CCE particle measurements that the very rapid initial Dst recovery in the great storm of February 1986 (minimum $D s t=-312 \mathrm{nT}$ ) resulted largely from the rapid loss of $\mathrm{O}^{+}$by means of charge exchange in the inner portion of the ring current $(L \sim 3-5)$. However, Fok et al. (1995) have pointed out by using their ring current decay model that, considering the energization of the continuously injected ions, other loss mechanisms in addition to the charge exchange losses are required to explain the rapid recovery of the Dst

Copy right(C) The Society of Geomagnetism and Earth, Planetary and Space Sciences (SGEPSS); The Seismological Society of Japan; The Volcanological Society of Japan; The Geodetic Society of Japan; The Japanese Society for Planetary Sciences. index. For instance, Kozyra et al. (1998) recently found that observed ion precipitation losses was of similar magnitude to the $\mathrm{O}^{+}$charge exchange losses during the early recovery of that great storm.

The results of particle tracing simulation suggest that untrapped charged particles which drift around the near-Earth magnetotail and then escape from the flank of the magnetopause into the magnetosheath would also affect largely on the initial rapid recovery of the Dst index (Takahashi and Iyemori, 1989; Takahashi et al., 1990a,b). We consider that the contribution of this charged particles flowing nightside and then escaping from the duskside flank and resultant daynight asymmetry of the ring current distribution have been less stressed and should be more thoroughly investigated in examining the storm-associated property of the Dst index. Actually, Roelof (1987) reported the presence of strong noon-midnight asymmetry (ratio $\gtrsim 20: 1$ ) in differential ion intensity in the ring current region $\left(3.0<r<5.0 \mathrm{R}_{\mathrm{E}}\right)$ during the main phase of a large magnetic storm (minimum Dst $=-241 \mathrm{nT}$ ) by using an image of energetic neutral atoms.

In this study, we have examined the disturbed magnetic field structure in the inner magnetosphere using the vector magnetic field data obtained by the ETS-VI satellite near the equatorial plane in the region between $\sim 3.0$ and 7.1 $\mathrm{R}_{\mathrm{E}}$ (geocentric distances in Earth radii). We will show nonnegligible influence of the nightside currents in the outer ring current-inner plasma sheet region $\left(5.6 \sim 7.2 \mathrm{R}_{\mathrm{E}}\right)$ on the rapid recovery of the Dst index.

\section{Data}

We have used the magnetic field data obtained by the ETSVI satellite near the equatorial plane at radial distances from 


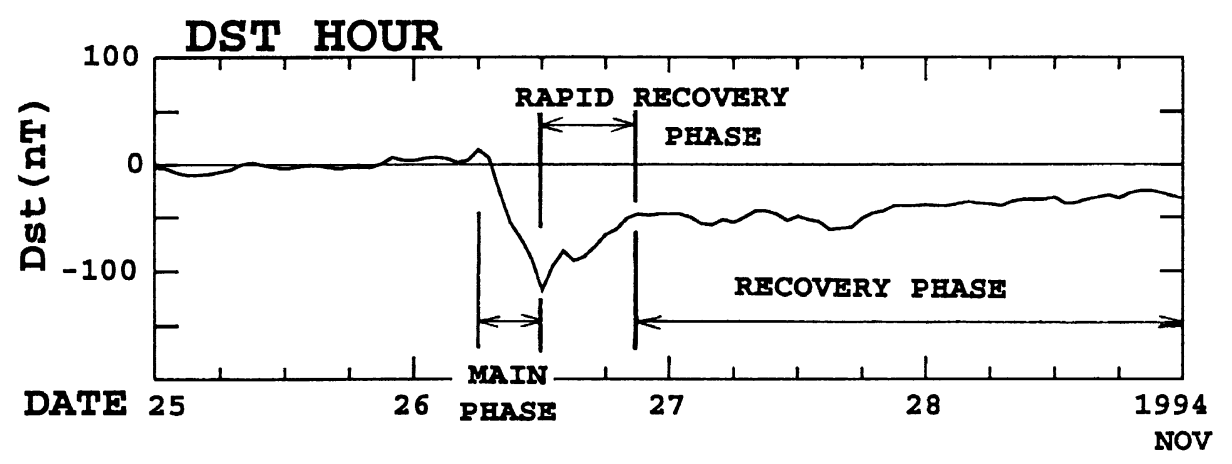

Fig. 1. A typical example of the Dst development during the geomagnetic storm on November 26, 1994. Each storm phase is indicated on the panel.

\section{ETS-VI ORBITS \\ PROJECTED ON \\ THE MAGNETIC EQUATORIAL PLANE}

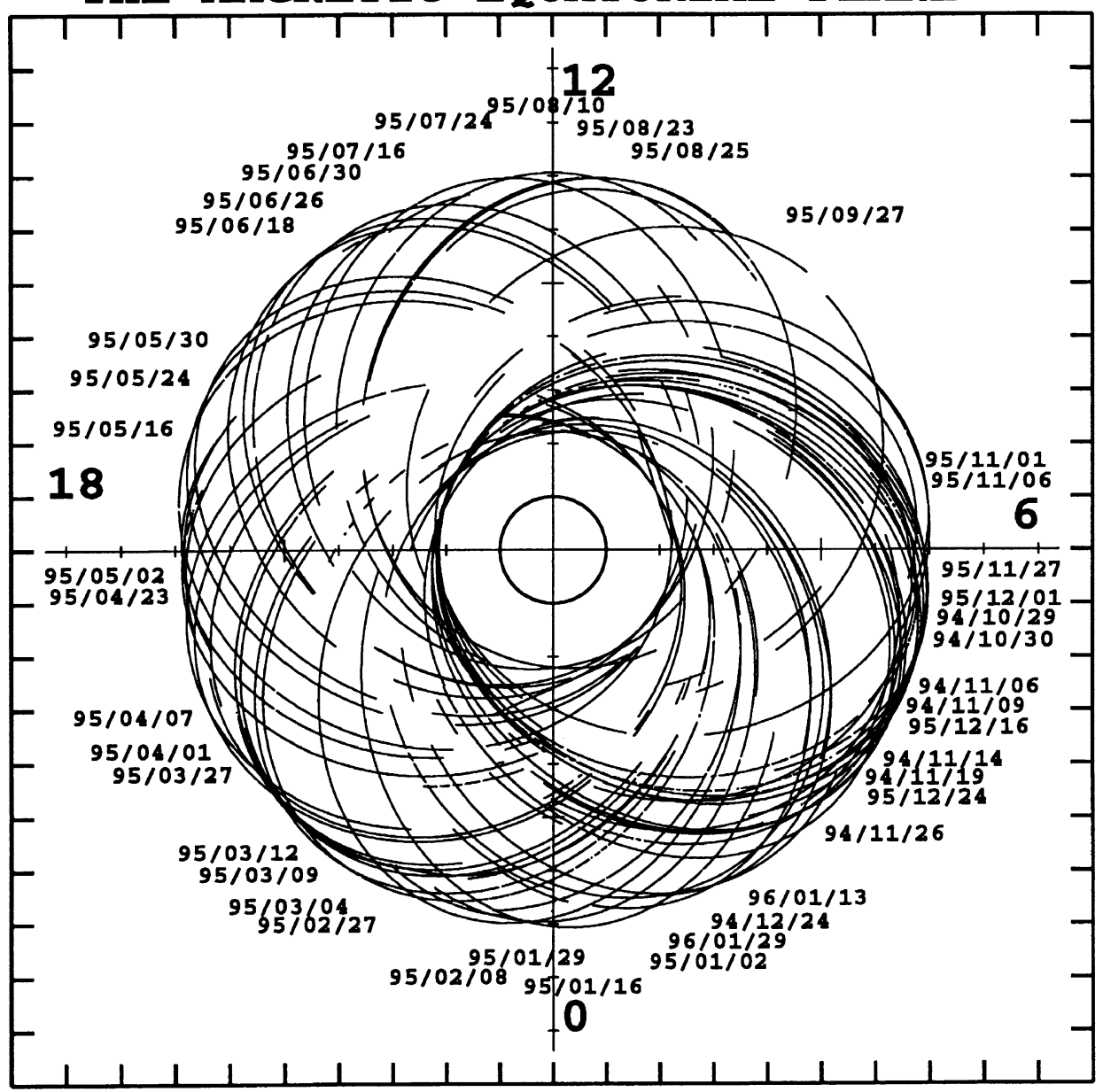

Fig. 2. Successive projections of the ETS-VI orbit on the geomagnetic dipole equatorial plane during the magnetic storms used in the present analysis. Only the dates and orbits of the first day at which each magnetic storm commenced are shown. 

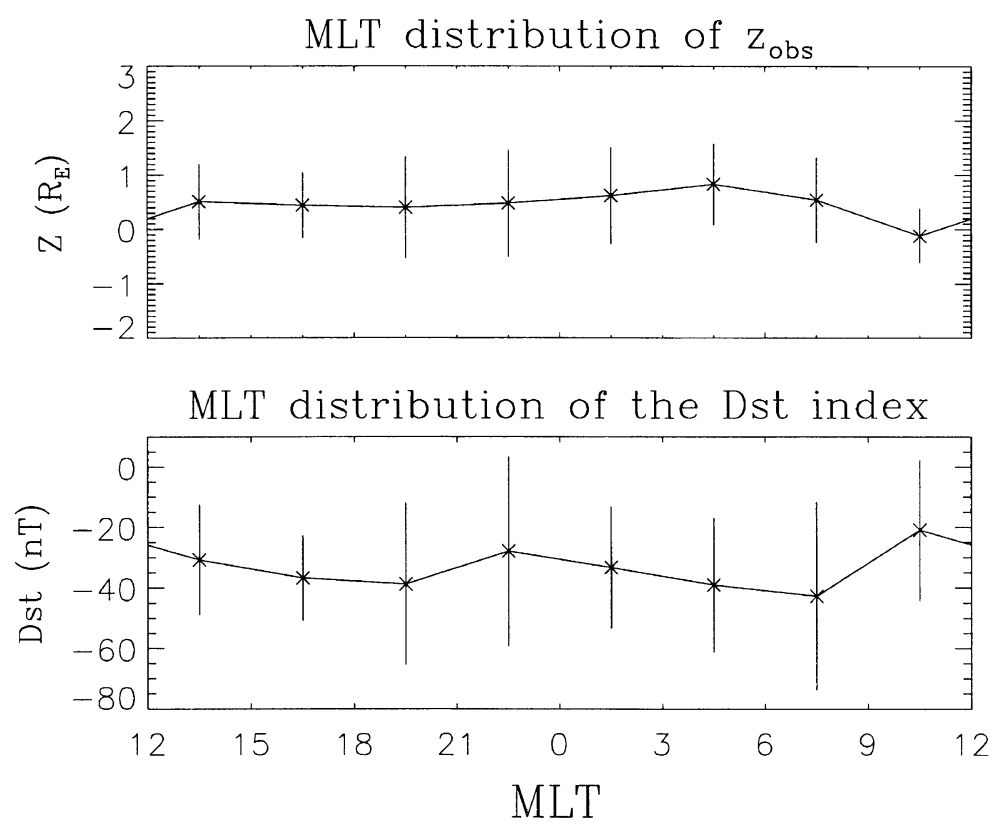

Fig. 3. Upper panel illustrates the magnetic local time distribution of the observed data-point locations from the dipole magnetic equator, $z_{\text {obs }}$ Vertical bar represents the standard deviation of $z_{\text {obs }}$ distribution at each MLT sector. In the lower panel, the relation between the data-point MLT locations and the Dst index at the time when observations were done is illustrated with standard deviations.

$\sim 3.0$ to $7.1 \mathrm{R}_{\mathrm{E}}$. The ETS-VI satellite had a near equatorial elliptical orbit with a perigee of $2.3 \mathrm{R}_{\mathrm{E}}$, an apogee of $7.1 \mathrm{R}_{\mathrm{E}}$, a $13.4^{\circ}$ inclination and a 14.4-hour orbital period, although, at first, it was intended to have a geosynchronous orbit. The location of apogee had moved from 05 MLT in October 1994, through midnight, after undergoing one and a half revolution around the Earth's dipole axis, to 16 MLT in July 1996 (Fig. 2). It covers almost all magnetic local time in the vicinity of the geomagnetic dipole equatorial plane. The ETS-VI magnetic field experiment includes a magnetometer (MAM) which consists of a triaxial fluxgate magnetometer mounted directly on the top of the $3 \mathrm{~m}$-height antenna tower (see, Nagai et al., 1996). The magnetometer has two switchable dynamic ranges: Range-L $( \pm 65536 \mathrm{nT})$ and Range-H $( \pm 256 \mathrm{nT})$ with resolutions $32 \mathrm{nT}$ and $0.125 \mathrm{nT}$, respectively (a 12-bit A/D converter is used). High resolution measurements (Range$\mathrm{H}$ ) are carried out over $\sim 11$ hours near apogee (at radial distances of $5.0 \sim 7.1 \mathrm{R}_{\mathrm{E}}$, and at $-25^{\circ} \sim 25^{\circ}$ magnetic latitudes), and measurements with Range- $\mathrm{L}$ mode are conducted near perigee. The time resolution of the magnetometer data is 3 seconds. The spacecraft field was evaluated by comparing magnetic field measurements on geomagnetically quiet days with the IGRF1990 model fields and the empirical fields produced by the quiet-time magnetospheric currents (Tsyganenko, 1987; Langel et al., 1981). This spacecraft field was subtracted from the magnetic field data obtained in the satellite reference frame.

In this study, field data are presented in the cylindrical magnetic coordinate system $(\rho, \phi, z)$ with the origin at the center of the Earth. In this coordinate system, the $z$ axis is antiparallel to the Earth's dipole, i.e., positive northward, $\rho$ is orthogonal to $z$ and is contained in the plane parallel to the geomagnetic equator, pointing radially outward, and the azimuthal angle $\phi$ points positive eastward.

The magnetic field data, from October 26, 1994 to July 5, 1996 , cover the 504-day period. To investigate the magnetic storm-associated disturbances, we have chosen 41 "typical" magnetic storms having minimum $D s t$ index below $-40 \mathrm{nT}$ (Fig. 2), and then classified them in the four storm phases using the Dst index: the main phase, the rapid recovery phase, the recovery phase, and the quiet period (Fig. 1). Here, "typical" means that the classification of the period into the four phases is rather easy for the storm. The end of the recovery phase is determined by using the criterion that both magnitude and variation of the Dst index reduce to nearly zero. The period with the Dst index being nearly zero which is obviously far from the storm periods and lasts at least a few days was selected as the quiet period. The total time of each phase period is $345.5,311,1613$, and 2088 hours with the average $D s t$ index being $-38.0,-49.0,-26.1$, and $-3.0 \mathrm{nT}$, respectively.

To investigate whether the data at the dayside and nightside have uniform distribution in Dst activity or not, we have examined the relation between the data-point locations and the Dst values at the time when observations were done. Also, the uniformity of satellite locations from the dipole equatorial plane, $z_{\mathrm{obs}}$, has been examined. Figure 3 illustrates MLT dependence of their distributions with standard deviations during the main phase. Considerably large standard deviation in the Dst distribution (lower panel) is partly caused by the fact that the Dst index changes from nearly zero to its minimum value during the main phase. Although the data points used in our study are sparse at the dayside during the main phase (and also during the rapid recovery phase), satellite locations from the equatorial plane are rather uniformly distributed with their averages near $z=0.5 \mathrm{R}_{\mathrm{E}}$ except for 

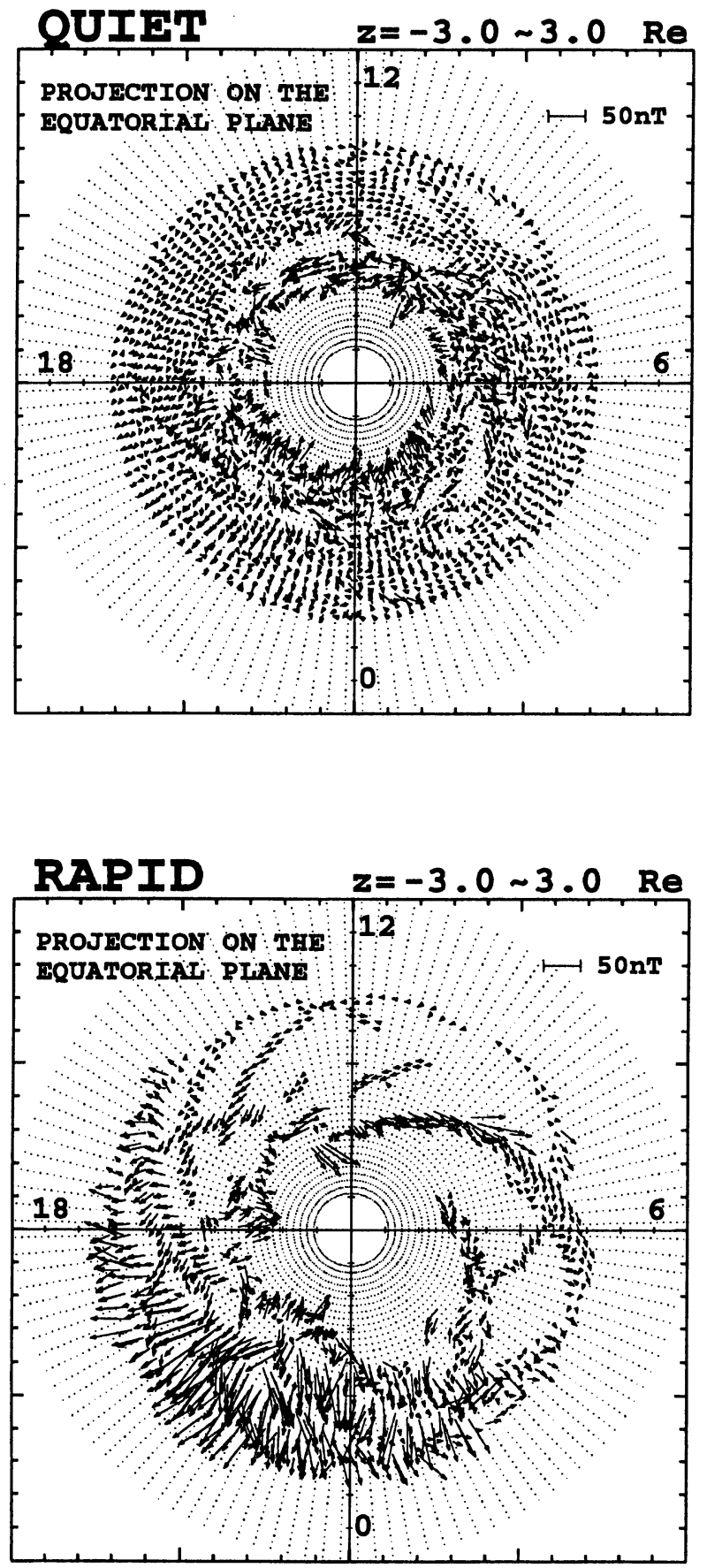
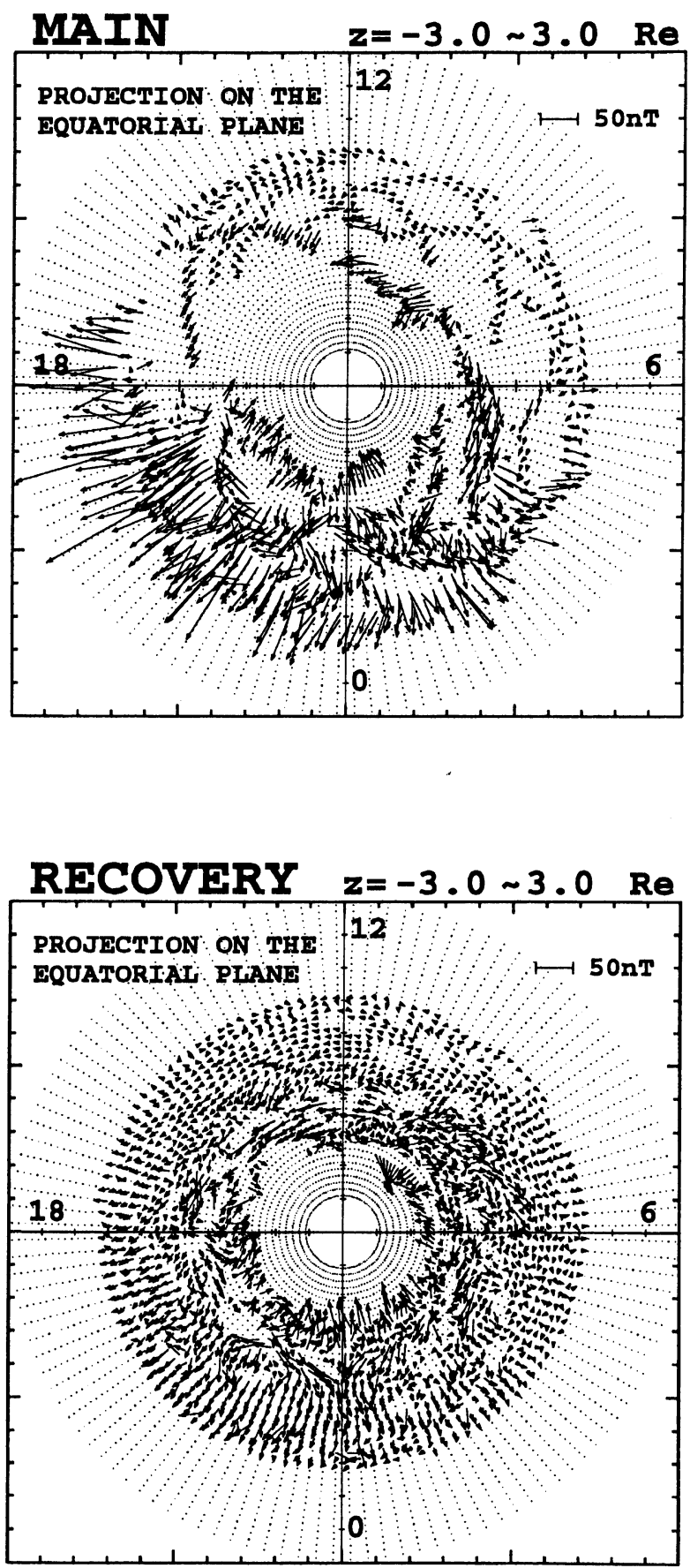

(a)

Fig. 4. Distributions of magnetic field disturbance on the equatorial plane during the quiet period (top left), the main phase (top right), the rapid recovery phase (bottom left), and the recovery phase (bottom right). (a) Each vector represents the radial and azimuthal perturbed field components, $\Delta B_{\rho}$ and $\Delta B_{\phi}$. (b) The radius of circle (the size of triangle) is proportional to the amplitude of the southward (northward) perturbed field component, $-\Delta B_{z}\left(\Delta B_{z}\right.$ ).

9-12 MLT sector. Dst activities also do not have apparent uneven distribution (in this case, relatively low Dst activity regions exist around 21-0 and 9-12 MLT sectors). However there exists rather large difference in each storm scale and it might cause a limitation of our analysis rather than their MLT distribution. This should be kept in mind. The MLT distributions for other phases are fairly uniform, and we omit to show them here.

\section{Magnetic Field Observations}

In this study, the ETS-VI magnetic field data from which the IGRF1990 model fields and the spacecraft field are subtracted are used. We denote these residual magnetic field components as $\Delta B_{\rho}, \Delta B_{\phi}$, and $\Delta B_{z}$ in the cylindrical magnetic coordinate system defined here. Figure 4(a) illustrates the projections of averaged residual magnetic field vectors on the $\rho-\phi$ plane, i.e., each vector represents $\Delta B_{\rho}$ and $\Delta B_{\phi}$, 

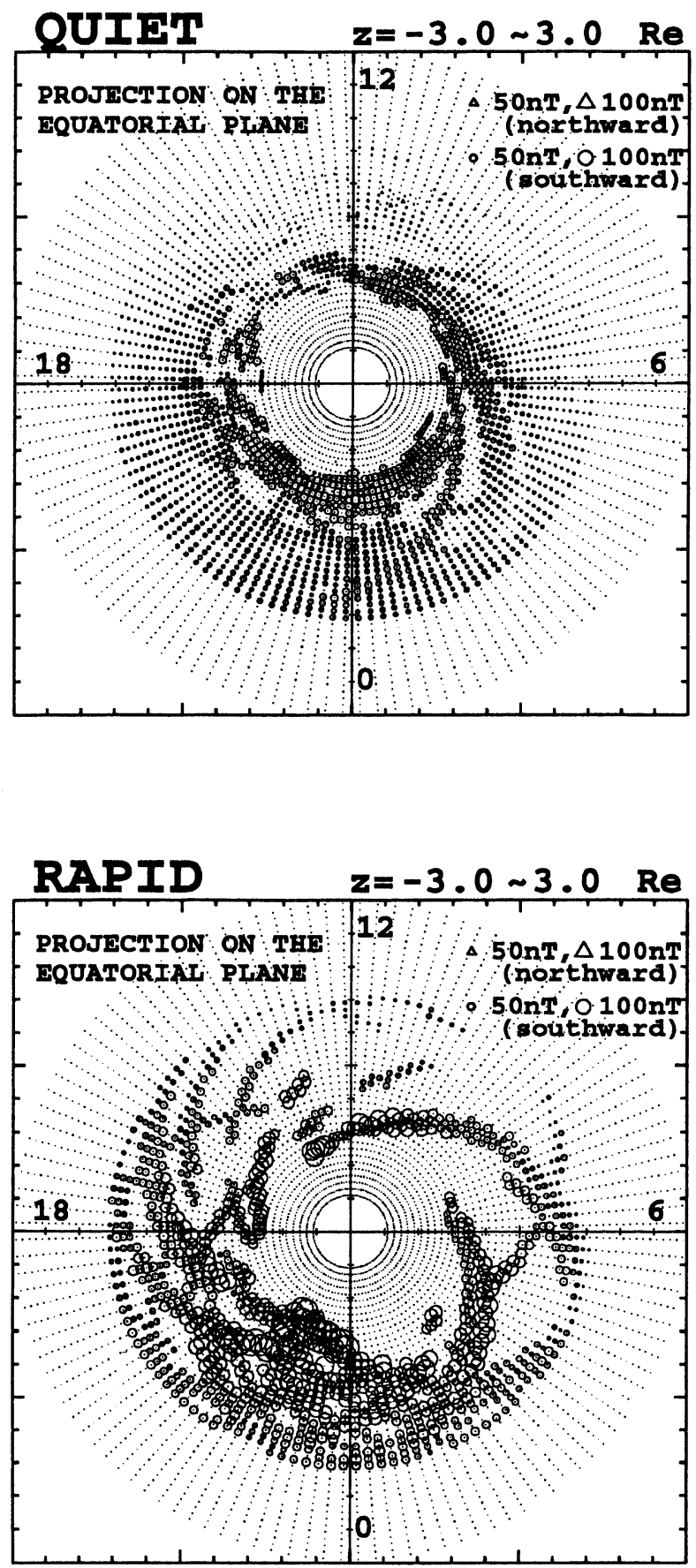
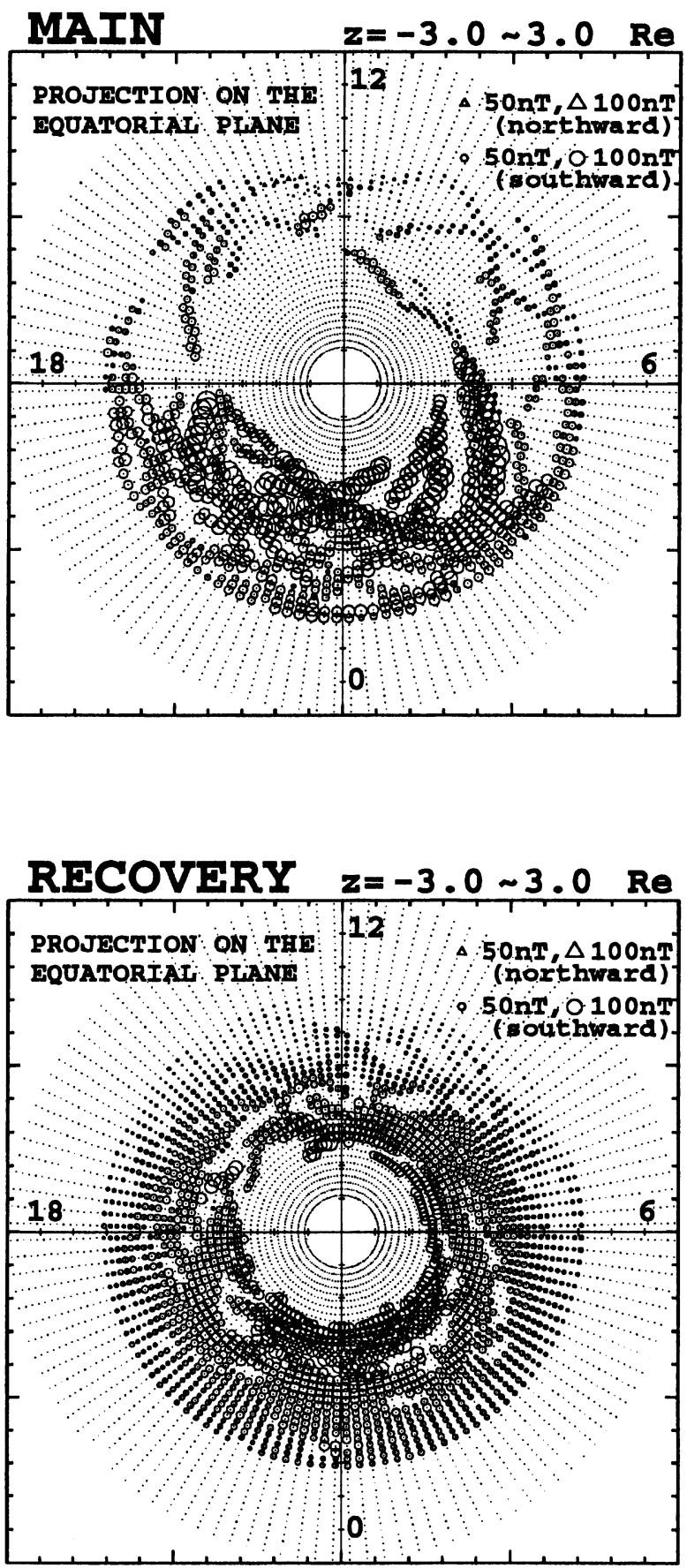

(b)

Fig. 4. (continued).

for the quiet period (top left), the main phase (top right), the rapid recovery phase (bottom left), and the recovery phase (bottom right). The other component, $\Delta B_{z}$, is also illustrated in Fig. 4(b) as a circle or a triangle. The radius of circle (the size of triangle) is proportional to the amplitude of the southward (northward) perturbed field component, $-\Delta B_{z}\left(\Delta B_{z}\right)$. To investigate average storm-associated magnetic field variations, perturbed field data are firstly divided into and then averaged within the $\rho-\phi-z$ bins of increments of $0.2 \mathrm{R}_{\mathrm{E}}, 0.2$ hour, and $0.2 \mathrm{R}_{\mathrm{E}}$, respectively. In Figs. 4(a) and 4(b) those averaged values are averaged again in the $z$ direction. There are almost no data beyond $z=-3.0 \sim 3.0 \mathrm{R}_{\mathrm{E}}$ due to the small inclination $\left(13.4^{\circ}\right)$ of the satellite orbit, and most of the data points concentrate within $z=-2.0 \sim 2.0 \mathrm{R}_{\mathrm{E}}$. It should be noted again that, although the high resolution magnetometer data (resolution is $0.125 \mathrm{nT}$ ) are available for radial distances of $\gtrsim 5 R_{E}$, there are only the magnetometer data with Range- $\mathrm{L}$ mode (resolution is $32 \mathrm{nT}$ ) below $\sim 5 \mathrm{R}_{\mathrm{E}}$. Assuming that the magnetospheric currents are flowing parallel to the geomagnetic equatorial plane and this current structure 


\section{$\triangle B Z$ MAIN}

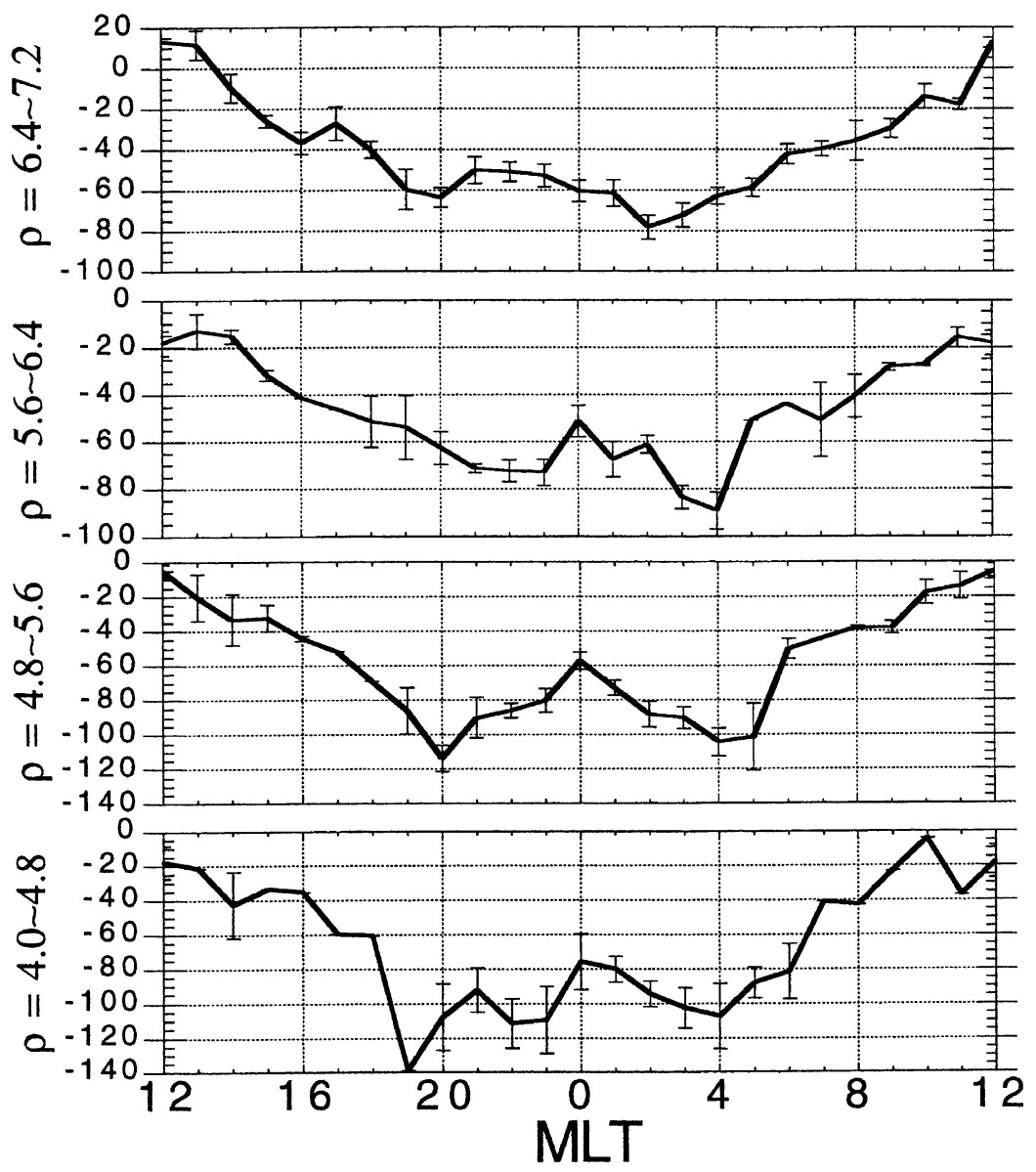

(a)

Fig. 5. The local time distributions of the southward component of perturbed magnetic fields with standard errors, which was observed at the different geocentric distances near the geomagnetic equator during the main phase (a), rapid recovery phase (b) and recovery phase (c).

has a north-south symmetry with respect to the equatorial plane, $\Delta B_{\rho}$ and $\Delta B_{\phi}$ above the equatorial plane (in most cases pointing radially inward) have the polarities opposite to the ones below the equatorial plane (mostly pointing outward). For the convenience of visualization, the perturbed fields above the dipole equatorial plane are set to have the same polarities of the ones below the equatorial plane, i.e., the polarities of $\Delta B_{\rho}$ and $\Delta B_{\phi}$ above the equatorial plane are reversed.

In the panels of the main and the rapid recovery phases of Figs. 4(a) and 4(b), clear day-night asymmetry of perturbed magnetic fields can be seen. It should be noted that this asymmetry of $\Delta \boldsymbol{B}$ is caused by the sum of the various magnetospheric current contributions in addition to the ring current contribution. In particular there are significant contributions to the asymmetry of $\Delta B_{z}$ from the magnetopause and the magnetotail currents, and each of them causes the noon-midnight asymmetry of about $30 \mathrm{nT}$ for $\Delta B_{z}$ distribution near geosynchronous altitudes (Tsyganenko, 1996). Although the asymmetry of $\Delta B_{\rho}$ is also influenced by the field aligned-currents and other current contributions, their intensities are relatively small ( $\sim$ a few nT) (Tsyganenko,
1996) and this asymmetry can be considered to be mainly caused by the azimuthal currents flowing near the equatorial plane. This suggests the existence of day-night asymmetry of the azimuthal currents during the storm time with larger intensities in the nightside region. During the main phase, the magnitude of $\Delta B_{\rho}$ near the midnight sector between 5.0 and $7.2 \mathrm{R}_{\mathrm{E}}$ are about 8 times greater than the magnitudes around noon. In order to examine the local time distribution of the northward component of perturbed magnetic fields, $\Delta B_{z}$, we exhibit their distributions observed at different distances between 4.0 and $7.2 \mathrm{R}_{\mathrm{E}}$ during the main phase, rapid recovery phase and recovery phase in Figs. 5(a), 5(b) and 5(c), respectively. During the main phase (Fig. 5(a)), the largest negative values for $\Delta B_{z}$ occur near the premidnight and postmidnignt sectors at every distance, with those magnitudes increasing for smaller $\rho$. The nightside relative bump which is about 8-hour-wide around $\sim 4.0 \mathrm{R}_{\mathrm{E}}$ and becomes narrow as $\rho$ increases and finally vanishes at $6.4 \sim 7.2 \mathrm{R}_{\mathrm{E}}$, suggests the existence of the current depression region around midnight sector, which would correspond to the "current trough" region in Nakai et al. (1997). The tendency of this relative bump is still seen during the rapid recovery phase, though 


\section{$\triangle B Z$ RAPID}

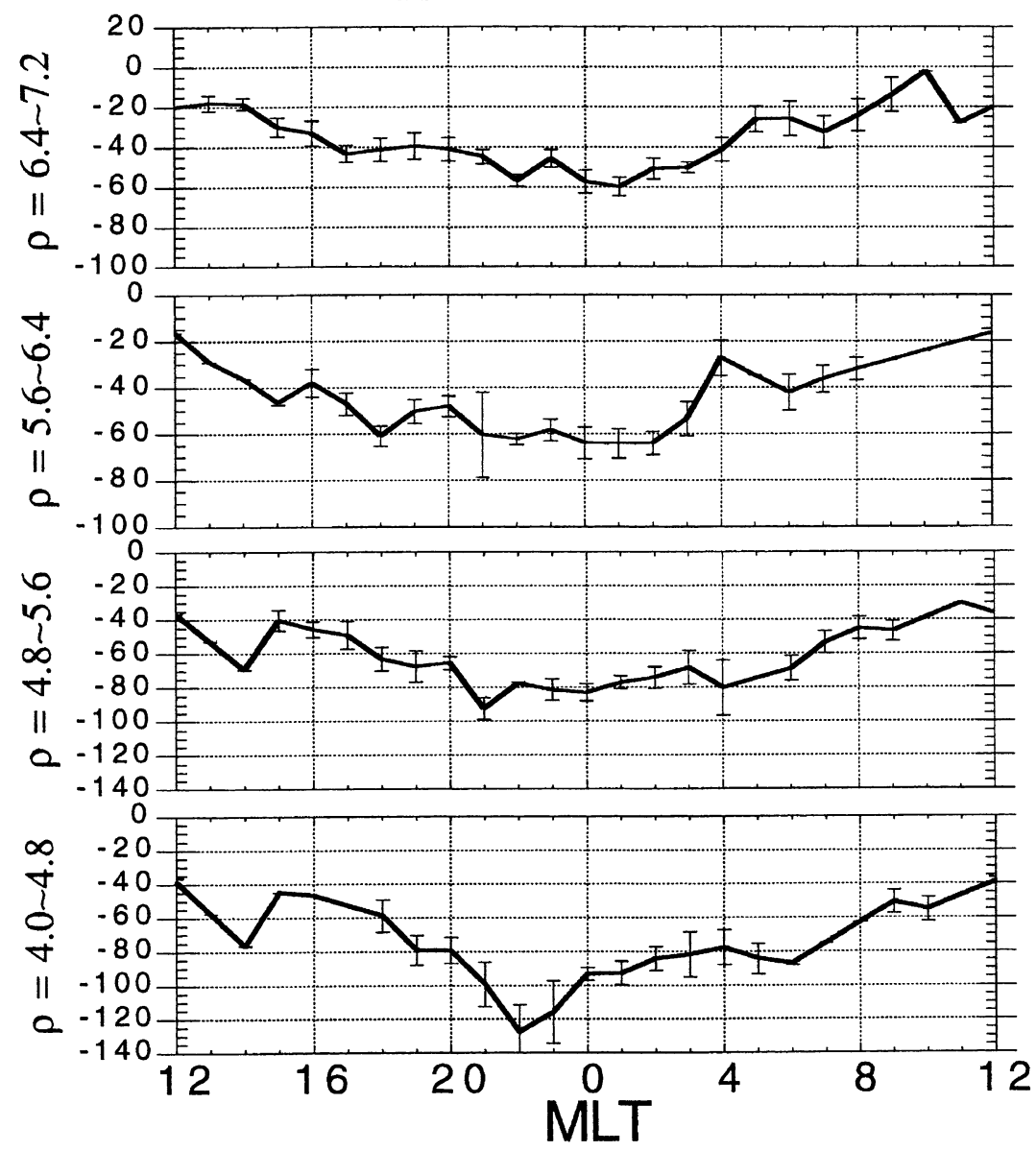

(b)

Fig. 5. (continued).

its magnitude is small and not clear (Fig. 5(b)). As is mentioned in the previous section, there is rather large difference in each storm event scale, and we have to be careful in interpreting these observed data. During the late recovery phase, $\Delta B_{z}$ distribution, in contrast, has a clear minimum near the midnight sector without any bumps (Fig. 5(c)), which is the same feature as seen in the figure 1 of Iijima et al. (1990).

During the main phase, radial and azimuthal magnetic field components are relatively large, and those amplitudes decrease as the storm phase evolves (Figs. 4(a) and 4(b)). This implies that the injected particles from the magnetotail form a sheet-like current structure in the early phase of a magnetic storm, because particles are accelerated in the perpendicular direction by the betatron acceleration, and then accelerated in the parallel direction by the mechanisms such as a waveparticle interaction and others in the later phase, forming torus-type current structure.

\section{Case Studies}

We have also examined each geomagnetic storm and compared magnetic field perturbations in the similar-scale storm events. Figure 6 illustrates the daily plots of the magnetic field perturbations during the early phase of the magnetic storms in the four similar-scale storm events: geomagnetic storms on May 24, 1995 (top left), on August 23, 1995 (top right), on March 12, 1995 (bottom left), and on November 19, 1994 (bottom right) with the minimum Dst being -65 , $-61,-70$, and $-56 \mathrm{nT}$, respectively. The hourly $D s t$ index as a function of time is shown in the lowest panel of each figure with one-minute resolution ASY/SYM indices. Magnetic field perturbations, $\Delta \boldsymbol{B}$, are illustrated via vectors and circles (or triangles) in the same way as Fig. 4. The satellite locations from the dipole equatorial plane were $z \sim 0$, $z \sim+0.5$, and $z \sim+1.5 \mathrm{R}_{\mathrm{E}}$ around their apogees for the top left, top right, and bottom left panels, respectively, and varied from $z \sim 0$ around the midnight sector to $z \sim+2 \mathrm{R}_{\mathrm{E}}$ near 6 MLT in the bottom right panel. These storm events and the variations of $\Delta \boldsymbol{B}$ are fairly typical except for top left one where the Dst index begins to develop at 19 UT on May 23 and reaches its minimum (-64 nT) at 23 UT of that day before the Dst development around 4 UT on May 24. Most of the storms including the bottom two show sudden development of the radial component of the magnetic field perturbations around the nightside region during the storm developing phase (compare these figures with that of quiet period in Fig. 4). In the bottom left panel, we can see that 
$\triangle$ Bz RECOVERY

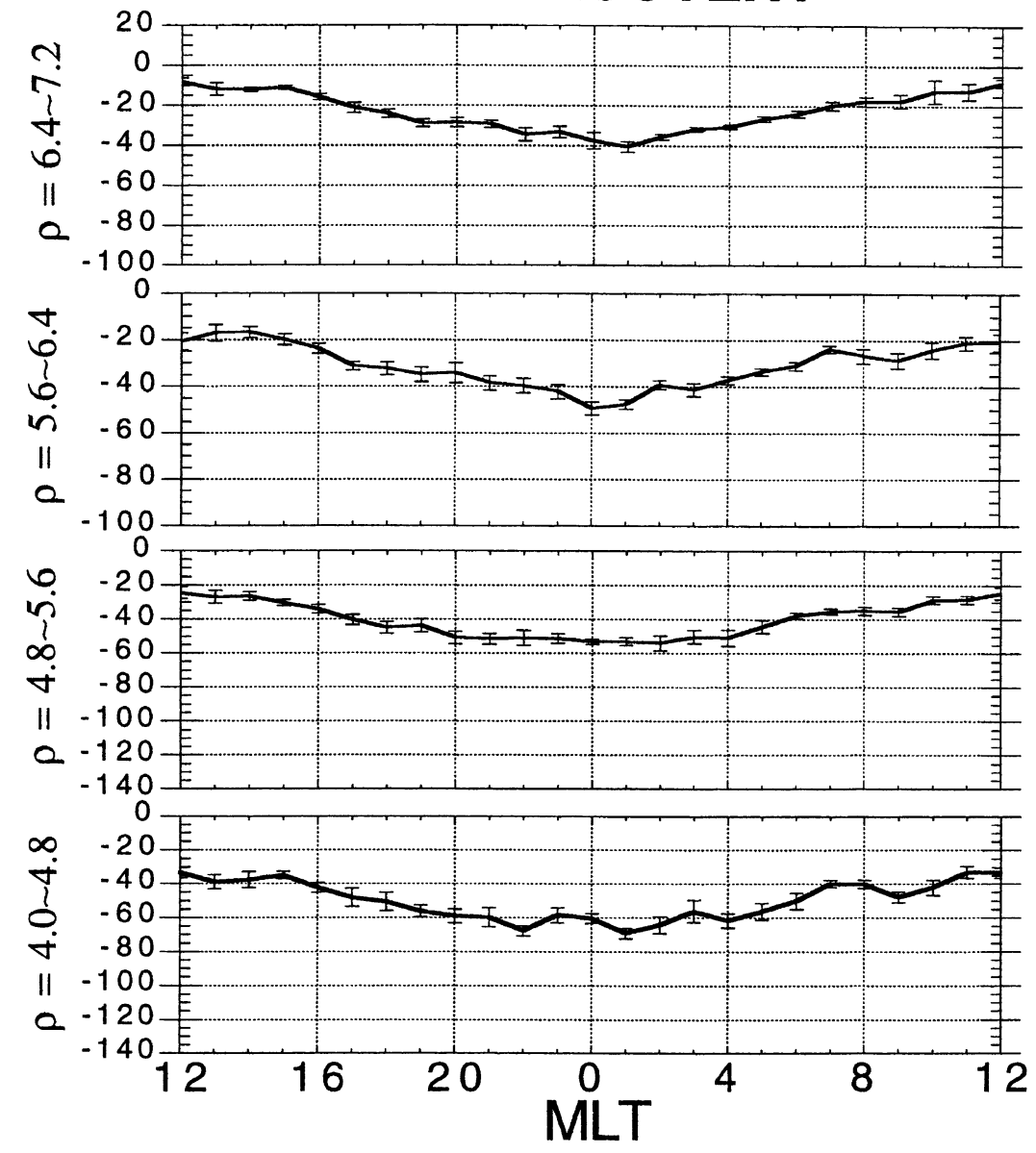

(c)

Fig. 5. (continued).

the radial perturbations rapidly decrease their intensities just in the middle of the rapid recovery phase. On the other hand, the dayside perturbations are very small throughout the storm-time (top right), whereas the top left panel shows noticeable perturbations (but small compared with the nightside ones) around the dayside region inside $\sim 6 \mathrm{R}_{\mathrm{E}}$ before the developing phase around $4 \mathrm{UT}$. We suppose that these perturbations are probably caused by the particles injected during the preceding main phase. They are quite rarely observed, and very small perturbations like the top right one are seen in any other storms. These four figures show the day-night asymmetry of the magnetic field disturbances and that the dayside perturbations are very small throughout the storm period even though, on some occasion during that period, injected particles would have reached the dayside region.

\section{Current Densities}

We have computed current densities in the inner magnetosphere with the data set of perturbed magnetic fields, $\Delta B_{\rho}$, $\Delta B_{\phi}$, and $\Delta B_{z}$, which are statistically determined at the $\rho$ $\phi-z$ grid points. In order to reduce noise level in taking curl of $\Delta \boldsymbol{B}$, perturbed fields are further averaged within the $\rho^{\prime}-\phi^{\prime}-z$ bins of increments of $0.8 \mathrm{R}_{\mathrm{E}}, 1.0$ hour, and $0.2 \mathrm{R}_{\mathrm{E}}$, respectively. The average numbers of storm events in the $0.8 \mathrm{R}_{\mathrm{E}} \times 1.0$ hour $\rho^{\prime}-\phi^{\prime}$ columns (obtained by integrating $\rho^{\prime}-\phi^{\prime}-z$ bins in the $z$ direction) are $13.6,5.1,4.2$, and 13.8 for the quiet, main, rapid recovery, and recovery phases, respectively, which are averaged over the region between 5.67.2 $\mathrm{R}_{\mathrm{E}}$ and 1800-2400-0600 MLT, i.e., the region used in the calculations below. We will denote this $\rho^{\prime}-\phi^{\prime}-z$ bins as $\rho-\phi-z$ bins in the following. The radial and azimuthal current densities, $j_{\rho}$ and $j_{\phi}$, are determined by using the Ampère's law under the assumptions that $j_{\rho}$ and $j_{\phi}$ are symmetrically distributed with respect to the geomagnetic equatorial plane and have no $z$-dependence near the equator. That is, we have first calculated $j_{\rho}$ between $\phi_{1}$ and $\phi_{2}$ and between $z_{0}(=0)$ and $z_{1}$, and $j_{\phi}$ between $\rho_{1}$ and $\rho_{2}$ and between $z_{0}$ and $z_{1}$ by using the following equations for every $\rho-\phi-z$ grid point:

$$
\begin{aligned}
& j_{\rho}=\frac{\int_{0}^{z_{1}} d z \int_{\phi_{1}}^{\phi_{2}} \rho d \phi(\nabla \times(\Delta \boldsymbol{B}))_{\rho}}{\mu_{0} \int_{0}^{z_{1}} d z \int_{\phi_{1}}^{\phi_{2}} \rho d \phi}, \\
& j_{\phi}=\frac{\int_{0}^{z_{1}} d z \int_{\rho_{1}}^{\rho_{2}} d \rho(\nabla \times(\Delta \boldsymbol{B}))_{\phi}}{\mu_{0} \int_{0}^{z_{1}} d z \int_{\rho_{1}}^{\rho_{2}} d \rho}
\end{aligned}
$$

with $\Delta B_{\rho}, \Delta B_{\phi}=0$ at $z=0$. In this study, $\phi_{2}-\phi_{1}$ is set equal to 1.0 hour, and $\rho_{2}-\rho_{1}$ is $0.8 \mathrm{R}_{\mathrm{E}}$. In the practical 

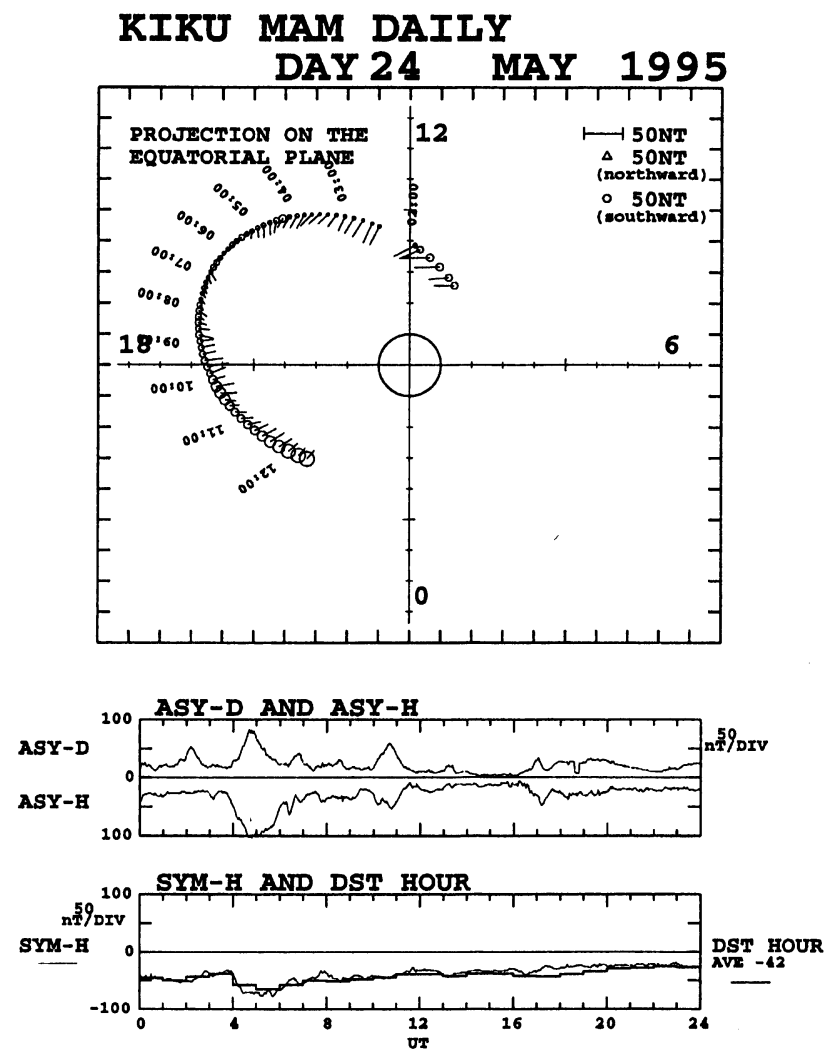

KIKU MAM DAILY DAY 12 MAR 1995
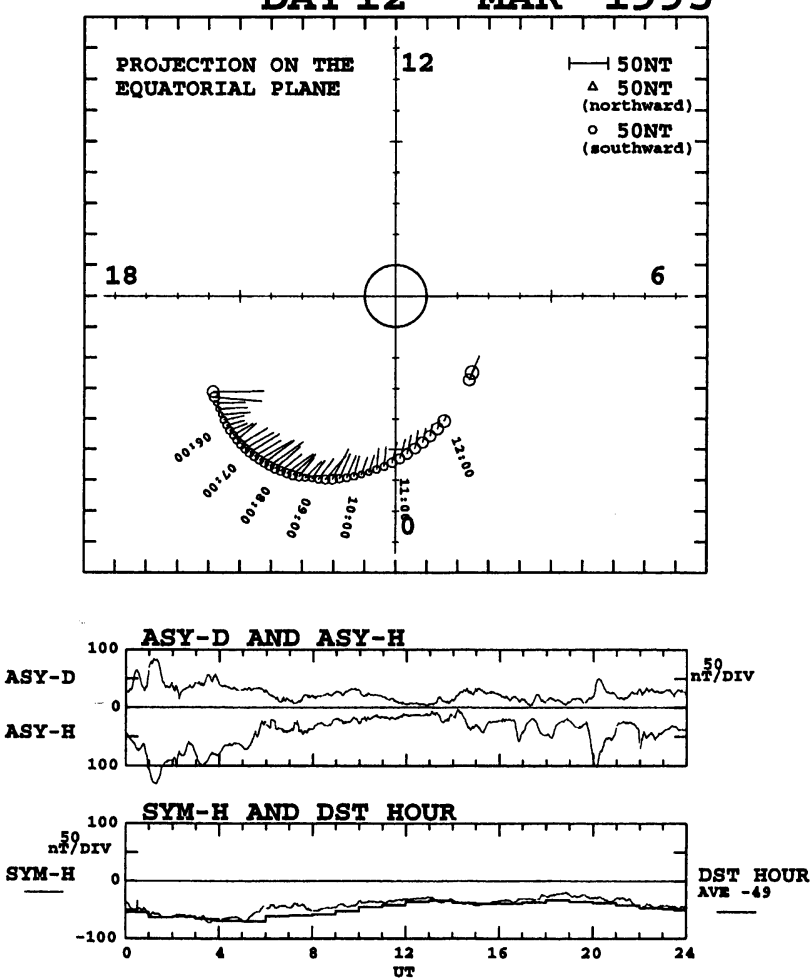

KIKU MAM DAILY DAY 23 AUG 1995
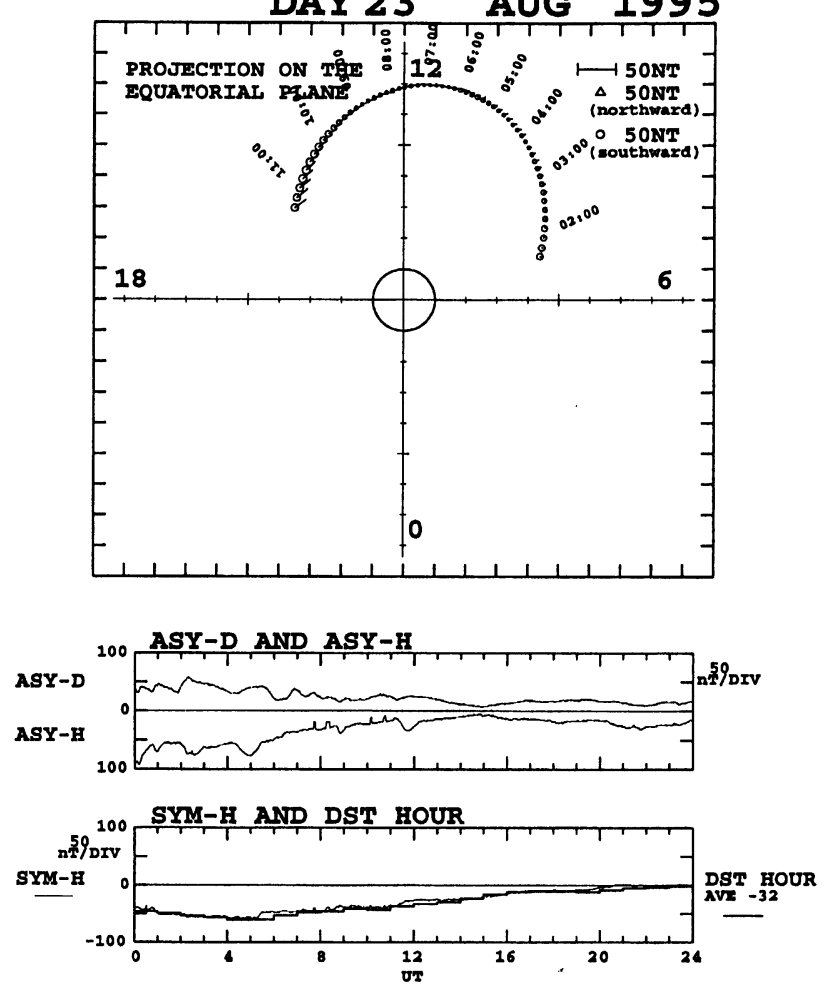

KIKU MAM DAILY DAY 19 NOV 1994
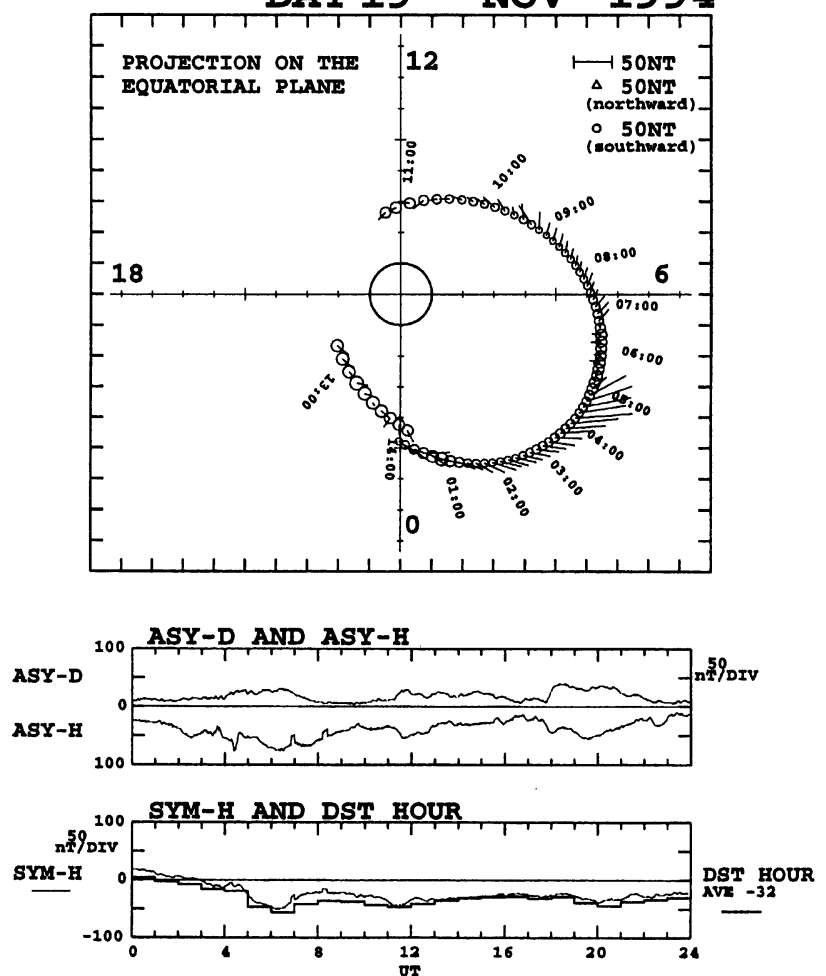

Fig. 6. Daily plots of the magnetic field perturbations during the early phase of the magnetic storms on May 24, 1995 (top left), on August 23 , 1995 (top right), on March 12, 1995 (bottom left), and on November 19, 1994 (bottom right) with the minimum Dst being -65, -61 , -70 , and $-56 \mathrm{nT}$, respectively. The hourly Dst index as a function of time is shown in the lowest panel of each figure with one-minute resolution ASY/SYM indices. Magnetic field perturbations, $\Delta \boldsymbol{B}$, are illustrated via vectors and circles (or triangles) in the same way as Fig. 4 . The times when measurements were carried out are also shown near the origin of the vectors. 

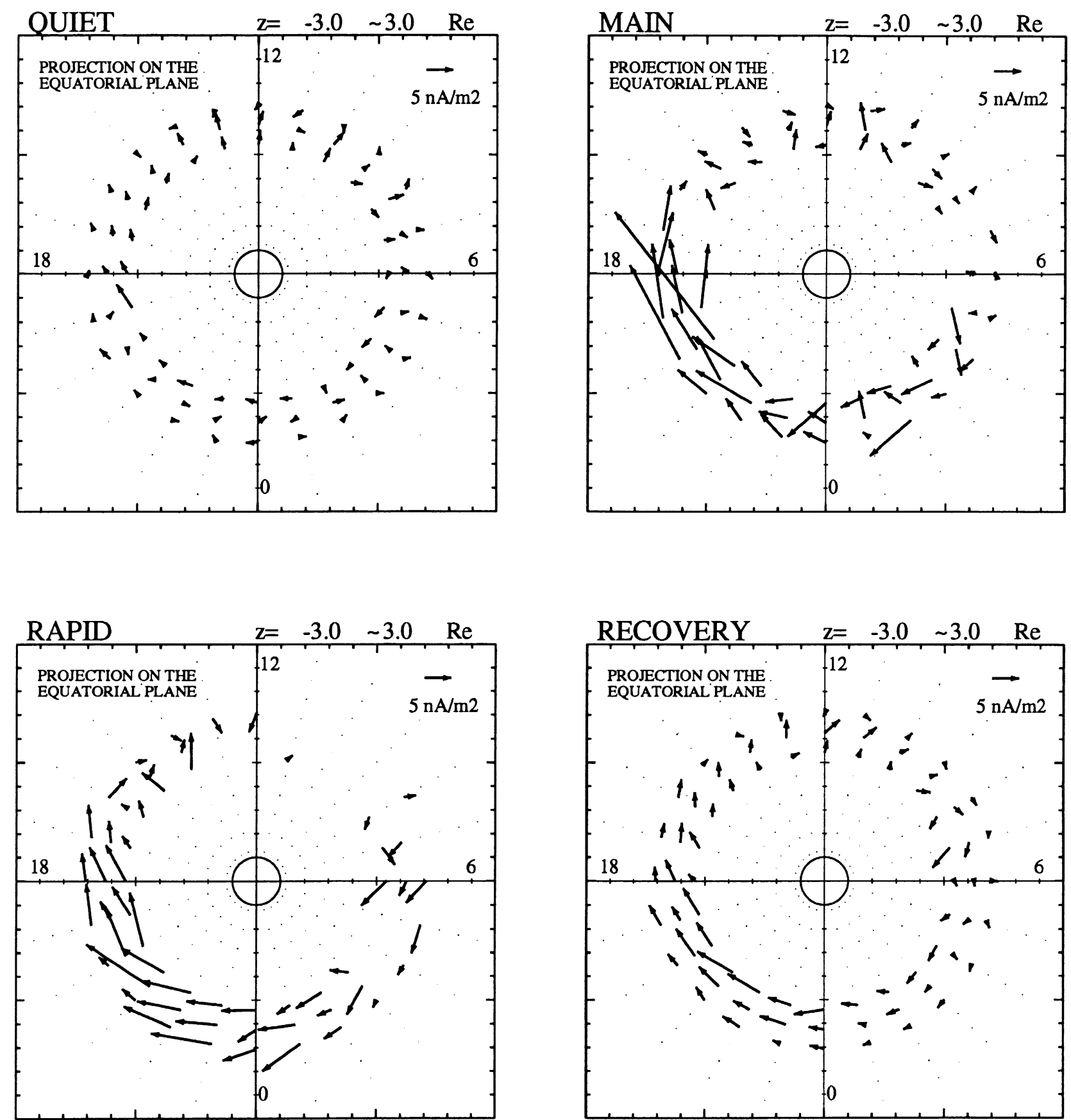

Fig. 7. Distributions of current density on the equatorial plane during the quiet period (top left), the main phase (top right), the rapid recovery phase (bottom left), and the recovery phase (bottom right). These current densities are computed by applying Eqs. $(2 \mathrm{a})$ and $(2 \mathrm{~b})$, assuming $\Delta B_{\rho}, \Delta B_{\phi}=0$ at $z=0$, and then averaged in the $z$ direction.

calculation, the binned magnetic field data are applied to the calculation as,

$$
\begin{aligned}
j_{\rho}= & \frac{1}{\mu_{0} z_{1} \rho\left(\phi_{2}-\phi_{1}\right)}\left[\Delta B_{z}\left(\phi_{2}\right) \cdot z_{1}\right. \\
& \left.-\Delta B_{z}\left(\phi_{1}\right) \cdot z_{1}-\Delta B_{\phi}\left(z_{1}\right) \cdot \rho\left(\phi_{2}-\phi_{1}\right)\right], \\
j_{\phi}= & \frac{1}{\mu_{0} z_{1}\left(\rho_{2}-\rho_{1}\right)}\left[-\Delta B_{z}\left(\rho_{2}\right) \cdot z_{1}\right. \\
& \left.+\Delta B_{z}\left(\rho_{1}\right) \cdot z_{1}+\Delta B_{\rho}\left(z_{1}\right) \cdot\left(\rho_{2}-\rho_{1}\right)\right] .
\end{aligned}
$$

We now obtained one or more current densities within a certain $\rho-\phi$ column according to the number of data points $\left(z_{1}\right)$ in the $z$ direction. Then these $j_{\rho}$ and $j_{\phi}$ are further averaged in the $z$ direction, and we have obtained the current densities, $j_{\rho}$ and $j_{\phi}$, for each $\rho-\phi$ grid point of increments of $0.8 \mathrm{R}_{\mathrm{E}}$ and 1.0 hour, respectively.

Figure 7 illustrates the calculated current densities, $j_{\rho}$ and $j_{\phi}$, for the quiet period (top left), the main phase (top right), the rapid recovery phase (bottom left) and the recovery phase (bottom right). The current densities around the nightside region have significant intensities during the main phase and then rapidly reduce their intensities as the storm phase evolves to the late recovery phase, while current den- 
sities do not change so much in the dayside hemisphere. We must annotate on some unusually large vectors around 20 MLT in the panel of the main phase. As mentioned above, we have calculated the current densities on the assumption that they have north-south symmetry, i.e., $\Delta B_{\rho}$ and $\Delta B_{\phi}$ vanish on the dipole equatorial plane. Because of the uncertainty of the location of the actual geomagnetic equator, observed $\Delta B_{\rho}$ and $\Delta B_{\phi}$ actually have non-zero values near the dipole equatorial plane. The unusually large current densities in the panel of the main phase are mainly produced by these non-zero $\Delta B_{\rho}$ and $\Delta B_{\phi}$ with small $z$ values in taking $\nabla \times(\Delta \boldsymbol{B})$. In particular, in the inner portion of the ring current region ( $\left.\lesssim 5 R_{E}\right)$, the very large currents could be a result of lack of the magnetic field observations with large $z$ values caused by the restriction of satellite orbits. Moreover, only the low-resolution data (resolution is $32 \mathrm{nT}$ ) are available inside $\sim 5 \mathrm{R}_{\mathrm{E}}$. So, the large current inside $\sim 5 \mathrm{R}_{\mathrm{E}}$ could be a ghost caused by the coarse resolution of Range-L mode, and we exhibit only the current densities above $5.0 \mathrm{R}_{\mathrm{E}}$ in Fig. 7.

Next, we have estimated the influence of the nightside magnetospheric currents on the Dst index. We have used the data set of the nightside current densities in the distant region between 5.6-7.2 $\mathrm{R}_{\mathrm{E}}$ and 1800-2400-0600 MLT. The current densities averaged over this region (which do not include the $z$ component contribution) are $1.2 \mathrm{nA} / \mathrm{m}^{2}$ for the quiet period, $6.8 \mathrm{nA} / \mathrm{m}^{2}$ for the main phase, $6.3 \mathrm{nA} / \mathrm{m}^{2}$ for the rapid recovery phase, and $2.6 \mathrm{nA} / \mathrm{m}^{2}$ for the recovery phase, respectively. Iijima et al. (1990) obtained the average current density in this region $\sim 4.0 \mathrm{nA} / \mathrm{m}^{2}$ with the data set during the disturbed conditions, defined by $2 \lesssim K p \lesssim 6$ and $-70 \lesssim D s t \lesssim-20 \mathrm{nT}$, with the average Dst being $-34 \mathrm{nT}$. In our study the observed average $D s t$ values are $-38.0 \mathrm{nT}$ for the main phase and $-26.1 \mathrm{nT}$ for the recovery phase, respectively. If we assume the current disk thickness to be 4.0 $\mathrm{R}_{\mathrm{E}}$ (Sugiura and Poros, 1973), the currents between 5.67.2 $\mathrm{R}_{\mathrm{E}}$ and 1800-2400-0600 MLT produce the contribution to the Dst index of $-12.0 \mathrm{nT}$ for the main phase and $-5.1 \mathrm{nT}$ for the recovery phase, respectively. The difference between them is $6.9 \mathrm{nT}$, while difference between the average values of the observed Dst index for the main phase and for the recovery phase is $11.9 \mathrm{nT}$. These values suggest that the influence from the nightside currents in the outer ring current region could be never neglected.

Our results were obtained without considering the $z$-dependence of the current densities and the temporal and seasonal variations of the location of the actual geomagnetic equator. To confirm that these results would not come from the effect of unusually large current densities near the dipole equatorial plane and the different tilt angle effect, we have performed the same calculation as was done above with neglecting the contribution of the currents near the equatorial plane. Inside $\sim 7 \mathrm{R}_{\mathrm{E}}$, the difference between the locations of the actual and the dipole magnetic equators at the midnight meridian is at the largest $\sim 1.0 \mathrm{R}_{\mathrm{E}}$ (e.g., figure A3 in Nakai et al., 1997). Therefore, we have calculated current densities using $\Delta B_{\rho}$ and $\Delta B_{\phi}$ with $z$ values greater than $1.0 \mathrm{R}_{\mathrm{E}}$, and consistent result is obtained. In this case, difference between the influences on the Dst index for the main phase and for the recovery phase is $4.8 \mathrm{nT}$, indicating that the unusually large currents and tilt angle effects do not drastically change our conclusion.

\section{Discussion}

In order to understand the mechanism of the growth and recovery of the ring current, charged particle motions in a realistic model magnetosphere (e.g., Mead and Fairfield model (1975)) were simulated using the guiding center approximation (Takahashi and Iyemori, 1989; Takahashi et al., 1990a,b). The simulation results show, during the stormtime, trajectories of the charged particles injected from the magnetotail region have significant day-night asymmetry caused by the asymmetry in gradient and curvature drifts that comes from the dayside compression and the nightside elongation of the magnetic field. For example, the protons injected from the nightside region whose initial kinetic energies are high (e.g., $\sim$ several tens $\mathrm{keV}$ ) drift around the nightside of the Earth and then escape from the duskside magnetopause to the magnetosheath (e.g., figure 5a in Takahashi and Iyemori, 1989). This is because the gradient and curvature drifts of the high energy protons are much faster than the $\boldsymbol{E} \times \boldsymbol{B}$ drift by the dawn-dusk electric field (the $\boldsymbol{E} \times \boldsymbol{B}$ drifts are independent of the particle energy, while the gradient and curvature drift velocities are proportional to the particle kinetic energy). Actually, such energetic escape ions have been observed by some satellites (e.g., Sibeck et al., 1987). On the other hand, the flow pattern of the particles whose initial energies are relatively low (e.g., $\sim$ several $\mathrm{keV}$ ) is only slightly asymmetric between the dayside and the nightside, and inner edge of the flow exists much closer to the Earth in comparison with the high energy one. The particles whose initial energies are relatively low are then trapped in the inner magnetosphere by expansion of the trapped (forbidden) region caused by the decrease of the dawn-dusk electric field, and drift around the Earth (Takahashi et al., 1990a).

This escapes of high energy protons from the duskside magnetopause could be important for the explanation of the asymmetry of magnetic disturbance fields near the geomagnetic equator. Moreover, they suggested that the two-step recovery of the Dst index might be explained by the superposition of the effects of "flow out" of high energy particles (or decrease of injection) and the loss process of trapped particles by charge exchange and wave-particle interaction.

The results of Hamilton et al. (1988) suggested that the very rapid initial $D s t$ recovery in the great storm (minimum $D s t=-312 \mathrm{nT}$ ) resulted largely from the rapid loss of $\mathrm{O}^{+}$ by means of charge exchange in the inner portion of the ring current. Although $\mathrm{H}^{+}$carries the majority of the energy during most of the storm, in the case of such a great storm, $\mathrm{O}^{+}$ dominates in the inner portion of the ring current $(L \sim 3-5)$ around the storm's maximum phase. They asserted that difference between the charge exchange lifetime $\sim 9$ hours for $\mathrm{O}^{+}$and $\sim 100$ hours for $\mathrm{H}^{+}$near their energy density peak in radial distance $L \sim 3$ and energy $\sim 100 \mathrm{keV}$ should cause the two-step recovery of the Dst index. This process may be certainly important. However, this result was obtained on the basis of the AMPTE/CCE particle measurements whose orbits have perigee at an altitude of $1100 \mathrm{~km}$ and apogee of 8.8 $\mathrm{R}_{\mathrm{E}}$ near $11 \mathrm{MLT}$ at that time, and did not consider the influences of the nightside particles in the outer ring current region. According to our calculation, the dayside currents in 
the outer ring current region, which were used in the analyses of Hamilton et al. (1988), Lui et al. (1987) and so on, do not much contribute to the initial rapid recovery of the $D s t$ index. Also, case studies of similar-scale storm events show the dayside magnetic field perturbations in the outer ring current region are much smaller than the nightside perturbations during the early phase of magnetic storms.

Our estimation of influences from the nightside currents in the outer ring current-inner plasma sheet region shows the importance of their contributions, and supports to include these currents into consideration in examining the cause of two-step recovery of the Dst index. These currents diminish their intensities considerably as the storm phase develops from the main phase to the recovery phase. What is more, Fig. 4(a) indicates that these currents would not flow into the dayside region. They are expected to escape from the flank region of the magnetopause into the magnetosheath according to the results of Takahashi and Iyemori (1989) and Takahashi et al. (1990a,b), or they are short-circuited by the field-aligned currents connected to the ionosphere. These results based on the observation suggest that the "flow out" loss of the particles in the outer ring current-inner plasma sheet region, cooperating with other loss mechanisms, would largely recover the Dst index during the rapid recovery phase. Then, we suppose, the Dst index recovers gradually by the loss of the inner portion of the ring current as is often said in the literatures (e.g., Fok et al., 1995).

\section{Summary}

To investigate the "two-step recovery" of the Dst index during the magnetic storm, we have examined the vector magnetic field data obtained by the ETS-VI satellite. We have classified them in the four storm phases using the Dst index and analyzed them statistically.

Our results are summarized as follows:

(1) Magnetic field perturbations during the storm-time exhibit clear day-night asymmetry with nightside magnitudes dominant. Although these perturbations are caused by the sum of the various magnetospheric current contributions, the asymmetry of $\Delta B_{\rho}$ distribution suggests that of azimuthal current distribution near the equatorial plane. We suppose that this nightside enhancement results from the storm-associated particle injections from the magnetotail region.

(2) During the main phase, southward perturbed field components have a relative bump in the nightside region between $\sim 2000$ and $\sim 0400$ MLT and between $\sim 4.0$ and $6.4 \mathrm{R}_{\mathrm{E}}$, which would correspond to the "current trough" region in Nakai et al. (1997).

(3) The initial rapid recovery of the Dst index is considerably influenced by the nightside currents flowing between 5.6-7.2 $\mathrm{R}_{\mathrm{E}}$ and 1800-2400-0600 MLT. These nightside currents in the outer ring current region have significant intensities during the main phase, and rapidly reduce their intensities with time. One noticeable feature is that they do not seem to flow into the dayside region. The constituents of these nightside currents are expected to flow out from the magnetosphere into the magnetosheath through the flank region of the magnetopause according to the results of the particle tracing simulations by Takahashi and Iyemori (1989) and Takahashi et al. (1990a,b), or they are short-circuited by the field-aligned currents connected to the ionosphere.

These results imply that the two-step recovery of the $D s t$ index cannot be explained by the rapid loss of the inner portion of the ring current only. The loss process of the nightside currents in the outer ring current region should be taken into consideration.

Acknowledgments. This work has been partly supported by the grant-in-aid for 1997 from ISAS and that from Japan Space Forum.

\section{References}

Akasofu, S.-I., S. Chapman, and D. Venkatesan, The main phase of great magnetic storms, J. Geophys. Res., 68, 3345-3350, 1963.

Fok, M.-C., T. E. Moore, J. U. Kozyra, G. C. Ho, and D. C. Hamilton, Threedimensional ring current decay model, J. Geophys. Res., 100, 9619-9632, 1995.

Hamilton, D. C., G. Gloeckler, F. M. Ipavich, W. Stüdemann, B. Wilken, and G. Kremser, Ring current development during the great geomagnetic storm of February 1986, J. Geophys. Res., 93, 14343-14355, 1988.

Iijima, T., T. A. Potemra, and L. J. Zanetti, Large-scale characteristics of magnetospheric equatorial currents, J. Geophys. Res., 95, 991-999, 1990.

Kozyra, J. U., M.-C. Fok, E. R. Sanchez, D. S. Evans, D. C. Hamilton, and A. F. Nagy, The role of precipitation losses in producing the rapid early recovery phase of the Great Magnetic Storm of February 1986, J Geophys. Res., 103, 6801-6814, 1998.

Langel, R., J. Berbert, T. Jennings, and R. Horner, Magsat data processing: A report for investigators, NASA Tech. Memo., 82160, 1981.

Lui, A. T. Y., R. W. McEntire, and S. M. Krimigis, Evolution of the ring current during two geomagnetic storms, J. Geophys. Res., 92, 7459-7470, 1987.

Mead, G. D. and D. H. Fairfield, A quantitative magnetospheric model derived from spacecraft magnetometer data, J. Geophys. Res., 80, 523$542,1975$.

Nagai, T., T. Ondoh, H. Matsumoto, T. Goka, T. Fukuda, M. Nosé, T. Iyemori, K. Takahashi, and S. Kokubun, ETS-VI magnetic field observations of the near-Earth magnetotail during substorms, J. Geomag. Geoelectr., 48, 741-748, 1996.

Nakai, H., Y. Kamide, and C. T. Russell, Statistical nature of the magnetotail current in the near-Earth region, J. Geophys. Res., 102, 9573-9586, 1997.

Roelof, E. C., Energetic neutral atom image of a storm-time ring current, Geophys. Res. Lett., 14, 652-655, 1987.

Sibeck, D. G., R. W. McEntire, A. T. Y. Lui, R. E. Lopez, S. M. Krimigis, R. B. Decker, L. J. Zanetti, and T. A. Potemra, Energetic magnetospheric ions at the dayside magnetopause: leakage or merging? J. Geophys. Res., 92, 12097-12114, 1987

Sugiura, M. and D. J. Poros, A magnetospheric field model incorporating the OGO 3 and 5 magnetic field observations, Planet. Space Sci., 35, 1763-1773, 1973.

Takahashi, S. and T. Iyemori, Three-dimensional tracing of charged particle trajectories in a realistic magnetospheric model, J. Geophys. Res., 94, 5505-5509, 1989

Takahashi, S., T. Iyemori, and M. Takeda, A simulation of the storm-time ring current, Planet. Space Sci., 38, 1133-1141, 1990a.

Takahashi, S., T. Iyemori, and M. Takeda, Ring current response to impulsive southward IMF: a cause of second development of the Dst index, $J$. Geomag. Geoelectr., 42, 1325-1331, 1990b.

Tsyganenko, N. A., Global quantitative models of the geomagnetic field in the cislunar magnetosphere for different disturbance levels, Planet. Space Sci., 35, 1347-1358, 1987.

Tsyganenko, N. A., Effects of the solar wind conditions on the global magnetospheric configuration as deduced from data-based field models, in Proc. of 3rd International Conference on Substorms (ICS-3), Versailles, France, 12-17 May 1996, ESA SP-389, 181-185, 1996.

N. Terada (e-mail: teradan@kugi.kyoto-u.ac.jp), T. Iyemori, M. Nosé, T. Nagai, H. Matsumoto, and T. Goka 